\title{
Structured-Light Based Sensing Using a Single Fixed Fringe Grating: Fringe Boundary Detection and 3-D Reconstruction
}

\author{
Jun Cheng, Chi-Kit Ronald Chung, Senior Member, IEEE, Edmund Y. Lam, Senior Member, IEEE, \\ Kenneth S. M. Fung, Fan Wang, and W. H. Leung
}

\begin{abstract}
Advanced electronic manufacturing requires the 3-D inspection of very small surfaces like the solder bumps on wafers for direct die-to-die bonding. Yet the microscopic size and highly specular and textureless nature of the surfaces make the task difficult. It is also demanded that the size of the entire inspection system be small so as to minimize restraint on the operation of the various moving parts involved in the manufacturing process. In this paper, we describe a new 3-D reconstruction mechanism for the task. The mechanism is based upon the well-known concept of structured-light projection, but adapted to a new configuration that owns a particularly small system size and operates in a different manner. Unlike the traditional mechanisms which involve an array of light sources that occupy a rather extended physical space, the proposed mechanism consists of only a single light source plus a binary grating for projecting binary pattern. To allow the projection at each position of the inspected surface to vary and form distinct binary code, the binary grating is shifted in space. In every shift, a separate image of the illuminated surface is taken. With the use of pattern projection, and of discrete coding instead of analog coding in the projection, issues like texture-absence, image saturation, and image noise of the inspected surfaces are much lessened. Experimental results on a variety of objects are presented to illustrate the effectiveness of this mechanism.
\end{abstract}

Index Terms-Binary pattern projection, fringe boundary detection, Ronchi pattern, specular surface, 3-D reconstruction, wafer bump.

\section{INTRODUCTION}

A S THE electronics industry advances toward manufacturing smaller, lighter, faster, and cheaper products, area array packages including ball grid array (BGA), chip-scale

Manuscript received July 20, 2007; revised September 6, 2007 and September 9,2007 . This work was supported in part by the Innovation and Technology Commission of Hong Kong Special Administrative Region, China, under an Innovation and Technology Fund with Project Code UIM/111. This work was recommended for publication by Associate Editor I. Fidan upon evaluation of the reviewers comments.

J. Cheng is with Shenzhen Institute of Advanced Integration Technology, Chinese Academy of Sciences/the Chinese University of Hong Kong, Shenzhen 518067, China, and also with the Chinese University of Hong Kong, Hong Kong, China. (e-mail: jun.cheng@siat.ac.cn).

C.-K. R. Chung is with the Department of Mechanical and Automation Engineering, The Chinese University of Hong Kong, Hong Kong, China. (e-mail: rchung@mae.cuhk.edu.hk).

E. Y. Lam is with the Department of Electrical and Electronic Engineering, University of Hong Kong, Hong Kong, China (e-mail: elam@eee.hku.hk).

K. S. M. Fung, F. Wang, and W. H. Leung are with ASM Assembly Automation, Ltd., Hong Kong, China (e-mail: smfung@asmpt.com; wang_fan04@yahoo.com.hk; whleung@asmpt.com).

Color versions of one or more of the figures in this paper are available online at http://ieeexplore.ieee.org.

Digital Object Identifier 10.1109/TEPM.2007.914209 packaging (CSP), flip chips, wafer bumping, and wafer-level packaging (WLP) are quickly becoming the focus of IC packaging technology. They could help improve device performance and manufacturability, and ultimately reduce cost. On the other hand, the shrinking dimensions of the devices impose more stringent requirements on process control and quality assurance. A particular challenge is the advancement of vision technique for inspecting accurately and efficiently the 3-D quality of microsurfaces like the solder bumps on wafers that are for direct die-to-die bonding. Specifically, the heights of, say, the solder bumps need to be measured and examined in three dimensions to ensure that they are within the specified bounds; for if they are not, the electrical connection could be compromised or even the dies can be damaged in the bonding process. There are already technologies for inspecting 3-D quality of solder bumps on printed circuit boards. However, the tiny size of the wafer bumps-which are typically of diameter 60 to $600 \mu \mathrm{m}$-make such technologies difficult.

There have been a few noncontact optical shape measurement methods proposed in the literature. They can be classified into two groups: scanning and the nonscanning techniques. The scanning techniques are represented by laser triangulation [1], [2] and confocal microscopy [3]-[5]. Both of them however require complex hardware to function. The measurement processes are also time consuming because they require one-dimensional, two-dimensional, or three-dimensional scanning to cover the entire surface of the object. Typical nonscanning techniques include conoscopy interferometry [6], [7], moiré interferometry [8], [9], and fringe pattern projection [10]-[21]. Conoscopy interferometry exploits the phenomenon of conoscopy - the interference behavior of doubly refractive crystals under convergent, polarized light. Moiré interferometry utilizes two initially identical gratings to create Moiré fringes. Surfaces of the inspected objects can be obtained by analyzing these fringes whose phases are proportional to range. Srinivasan et al. [20], [22] and Halioua and Liu [15] projected a periodical sinusoidal pattern onto the inspected surface $m$ times by shifting $1 / m$ of the period each time. For each pixel of the captured gray-level image, the associated phase of the first periodic pattern projected to the corresponding surface can be calculated from the $m$ intensity values at the pixel position in the image sequence grabbed across the phase shift. Once the phase of the pixel is known, the depth of the surface point can be precisely calculated. Wust and Capson [21] improved the mechanism. The proposed pattern consists of the superimposition of three periodical sinusoids instead of 


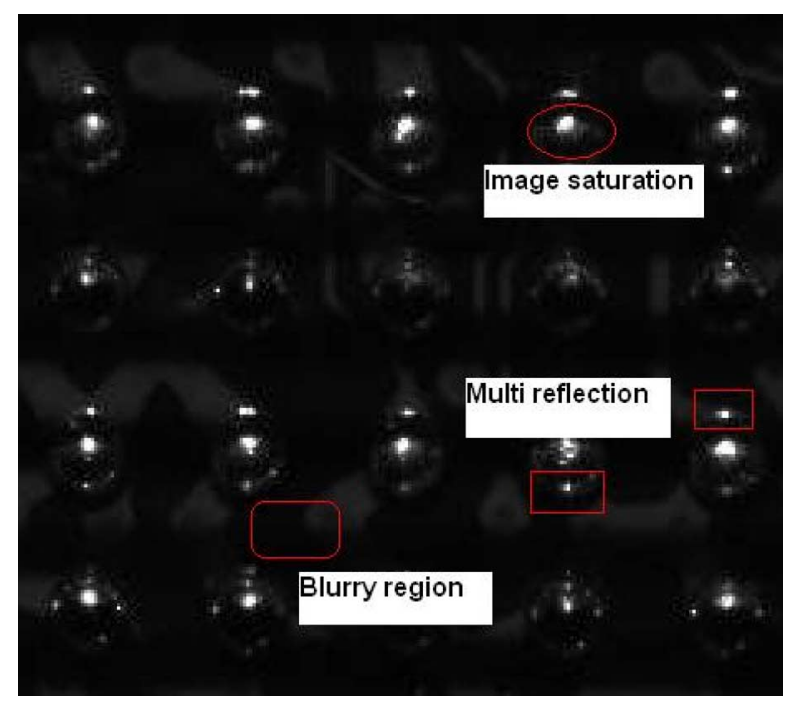

Fig. 1. Typical image of a wafer captured by analog pattern projection system, which displays the problems of image saturation, multireflections, and intensity blur or dilution.

one periodical sinusoid. Each sinusoid is associated with each primary color (red, green, and blue). The green's sinusoid is shifted $90^{\circ}$ with respect to the red, and the blue is shifted $90^{\circ}$ with respect to the green. This way, the phase of each pixel can be obtained from only one image. In brief, Moiré interferometry and pattern projection with phase shifting are about projecting a light pattern and capturing image of the illuminated scene from one or more points of view. Therefore, they have the advantages of 1) fast speed since they do not require scanning over the entire object surface, and 2) relatively high measurement resolution for they can accommodate phase shifting techniques.

Recently, Gühring [14] pointed out that phase shifting has a series of drawbacks: 1) the phase cannot be recovered precisely in dealing with surfaces with inhomogeneous reflectance function; and 2) intensity values at a pixel are influenced by those of its neighbors. The main limitation is that they obtain 3-D information based on analyzing gray-level fringes on the surface. Therefore, they suffer from the problems of image brightness saturation and high sensitivity to noise.

For illustration, we show in Fig. 1 the image of a wafer illuminated by gray-level pattern projection. In the image, it could be observed that regions around the solder bumps' top generally have their intensity values saturated because of the specular nature of the metallic bumps. Some regions on the substrate surface are however blurred, for the substrate is half-transparent and thus light could penetrate it partially, which leads to some light reflected from the surface and some from beneath it and therefore as a whole a dilution of the gray-level intensities. Besides, because the shape of every bump is hemispherical, light projected onto bumps could be reflected and illuminate other regions, and such multireflections, which are observable in Fig. 1 as those small bright dots, could greatly disturb the gray-level intensity values. All the above limit the accuracy of reconstructing the surface profile by the use of gray-level intensities, and in turn by the phase shifting mechanism.
One way to counteract image noise and gray-level saturation and alike problems is to replace the analog signals by discrete ones like the binary signals. Binary pattern projection and imaging for 3-D reconstruction is not a new concept. It has been explored under the name of structured light-based 3-D reconstruction. Posdamer and Altschuler [19], and Altschuler, Altschuler, and Taboada [10], [11] proposed a pattern composed of a dot matrix of $n \times n$ binary light beams. Each column of the pattern can be independently controlled to be lighted or obscured, thus equipping the illuminated position on the target surface a binary code either 1 or 0 . By projecting a sequence of $n$ patterns, they could encode $2^{n}$ stripes whose codewords are the sequence of $0 s$ and $1 s$ obtained from the $n$ patterns. Inokuchi, Sato, and Matsuda [18] furthered this encoding mechanism and proposed changing the binary codification to a Gray codification, which is more robust against noise. Caspi et al. [13] proposed a multilevel Gray code based on color. Pan [23] presented a color-coded binary fringe projection method that uses a combination of three primary colors to code the light patterns for 3-D shape measurement. However, because the monochrome CCD has higher quality and is more stable than color $\mathrm{CCD}$, the industry often prefers to use monochrome CCD over color CCD. Besides, the natural color of the substrate surface may cause confusion in the recognition of the projected color fringes.

With the illumination made binary or discrete, the problems of image brightness saturation and sensitivity to noise could be relieved, since only a bright or dark value instead of the absolute gray level needs be identified. By varying the binary pattern a number of times, each time capturing a distinct image of the illuminated surface, one could attach each position on the illuminated surface with a binary code in the sequence of captured images. By the use of suitable variations of the pattern, the binary code could even be made unique for each surface position. With such binary codes, position correspondences between the image plane and the projection panel (where the pattern is created in the projector) could be easily established. 3-D reconstruction is then possible on these coded points via triangulation.

As outlined previously, the traditional setup of the idea incorporates an array of light sources like the LCD panel. Such a device is however not suitable for inspection in VLSI manufacturing. The reason is, for the patterned light to cover a significant area of each microsurface (and thus allow the surface to be reconstructed more fully in 3-D), the light sources together are required to span an extended physical space, as illustrated by Fig. 2(a). That is undesirable as it could hinder the operation of the various moving parts that are involved in wafer processing.

This paper describes a new 3-D reconstruction mechanism that is designed with the goal of having a system size small enough not to hinder wafer processing. The mechanism is still based upon the principle of structured light, but adapted from the composition of an array of light sources to one with only a single light source [24]. The idea is to use a single light source together with a Ronchi grating (a binary grating consisting of alternate dark and transparent fringes) to allow a discrete pattern to be projected onto the inspected surface. As illustrated by Fig. 2(b), since only a single light source is used, the whole system is divergent as opposed to convergent in the configuration, occupying only a much smaller physical size. As in the 


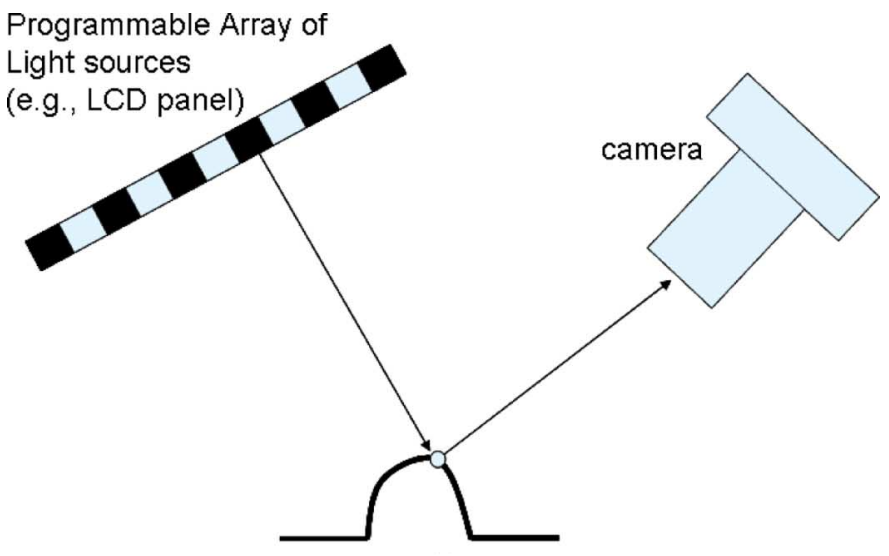

(a)

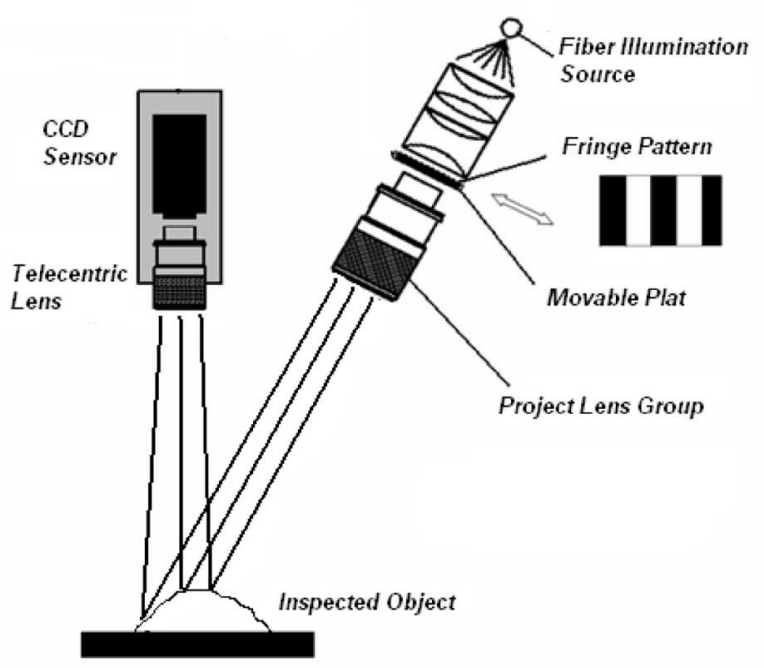

(b)

Fig. 2. Required physical space of (a) traditional structured light system that consists of multiple light sources, which corresponds to a convergent configuration. (b) Proposed system that consists of a single light source and a fringe grating, which corresponds to a divergent configuration.

traditional setup, a camera is also needed to capture image data of the illuminated surface. The camera is positioned at a distance from the light source so as to introduce enough baseline between the projection center and imaging center for accurate $3-\mathrm{D}$ reconstruction via triangulation.

New challenges come along with new design. The projection at any position of the inspection surface needs be varied, or else a distinct binary code for each position of the inspected surface could not be established. The traditional setup has control over each element of the projection panel (where the pattern is created), and does it simply by turning it on or off (in the base of binary pattern projection). In our system, we have a fixed pattern defined by the Ronchi grating, and we cannot possibly vary the pattern. How to vary the projection with only a fixed grating in hand, with the objective of equipping each position of the inspected surface with a distinct binary code, is an issue that comes with the modified setup of a structured light system.

We tackle the issue in the following way. We shift the Ronchi grating physically in space relative to the stationary light source and camera and everything else. In each shift, we take a separate image of the illuminated surface. This way, a series of dark or bright intensities over each scene position (which is associated with a particular image position, as both the camera and the inspected surface are made standstill in the entire process) are made available over the image sequence, and such intensity values together form the distinct binary code for that particular 3-D position. With enough shifting steps and image captures of the illuminated scene, the binary code for each image position of the associated 3-D position can even be made unique. Such binary codes will enable correspondence between positions on the image plane, and positions on the grating surface will be readily established, and thereby allowing 3-D structure of the illuminated scene to be reconstructed through a process similar to triangulation of stereo vision.

As pointed out earlier, since both the projected and perceived information are presumably binary, problems like image saturation and specular nature of the imaged surface could be much lessened. The use of pattern projection also allow textureless surfaces to be reconstructed. A number of complicating issues are thus tackled or reduced. Through theoretical analysis and empirical experiments on synthetic and real image data, this paper will show that the mechanism does hold a number of advantages over existing approaches.

However, with the twist of the structured light-based operation in the proposed mechanism, a number of challenging design issues arise that traditional structured light-based systems do not have. An obvious one is what the Ronchi grating's fringe pattern and the shifting steps of it should be. While in the traditional structured light-based setting, individual pattern elements could be changed independently of the others, which means the binary strings associated with the various image positions over the image sequence are entirely independent of one another, it is not the case in the proposed mechanism. In the proposed mechanism, the 0 -to- 1 or 1 -to- 0 value changes of the individual pattern elements are brought about by physical shifts of the Ronchi grating in space and are thus interrelated. The interrelationship is related to the fringe pattern on the Ronchi grating as well as to the sequence of shifting steps, and could affect how distinct the binary strings over the various image positions are from one another. In other words, a different fringe pattern and different shifting sequence could bring about less or more image snapshots of the illuminated scene (and thus shorter or longer binary strings) in the process of ensuring that the binary codes of the inspected positions are all unique.

The above design issue is however related not to the workability of the proposed mechanism but only to how many images are minimally needed in the processing and has been partially addressed in [25]. In this paper, we shall assume that we have a regular periodic fringe pattern on the grating, uniform-step shifting steps of the grating, and we take enough images to make the binary code at each image position distinct enough for establishing correspondences.

This paper aims at addressing the following two even more fundamental issues of it.

One fundamental issue is that, though intended to be binary information, the image data captured by commercially available cameras are inevitably gray level. In this paper, we describe a scheme of detecting the fringe boundaries in the image data (or equivalently speaking binarizing the image data) that is based 
upon the time profile of the intensity values at every image position, as opposed to upon the spatial gradients as practiced by most of the existing binarization mechanisms. Both theoretical analysis and experimental results show that the new scheme has advantages, especially upon scenes that have in the reflectance property a large variance ranging from the specular extreme (like over the metallic solder bumps) to the diffuse extreme (like over the nonmetallic wafer substrate surface).

This paper also provides a scheme of 3-D reconstruction that does not require explicit triangulation and correspondence between the image plane and the plane of the fringe grating. Instead, the use of a reference plane with an accompanying calibration step is exploited for the purpose.

The organization of the presentation is as follows. In Section II, we give an overview of the proposed binary projection system and describe how a 3-D structure can be recovered by it. In Section III, we show experimental results that demonstrate the feasibility of proposed approach. Concluding remarks are presented in Section IV.

\section{RONCHI GRATING-BASED APPROACH}

Continuous grating approach (e.g., sinusoidal grating method) uses gray-level intensities in image data to determine phase displacement and then surface profile. Because of the dependence on the absolute value of the perceived intensity, the approach suffers from problems like brightness saturation, multireflections, and noise or disturbance to gray-level brightness.

Should the continuous fringe pattern be replaced by a Ronchi (i.e., light-passing and light-blocking) pattern, a distinct binary code could be given to each surface point through a number of physical shifts of the fringe grating and image captures of the illuminated surface. Such binary codes are observable from both the plane of fringe grating at the illumination end and the image plane at the sensing end. Because of the preservation of the binary codes at both the illumination and the sensing ends, position correspondences could be established between the fringe grating plane and the image plane. In principle, such correspondences, each induced by a surface point on the illuminated surface, would allow the depth of the surface points and in turn the 3-D shape of the illuminated surface to be reconstructed.

Below we address three important issues, namely 1) how the binary codes are made observable from the image data, 2) how the fringe boundaries in the image data are detected, and in so doing having the image data binarized properly, so as to let the binary codes be directly observable, and 3) how 3-D structure of the illuminated surface could be recovered by the use of a reference plane that makes explicit triangulation unnecessary and system calibration much simplified.

\section{A. Binary Code Establishment}

For simplicity, suppose that the optical axes of the camera and illumination are aligned so that they are coplanar; here we define the optical axis of the illumination as the line that embeds the source (point or parallel) of illumination and that is perpendicular to the plane of the fringe grating. We shall refer to the plane that contains both the two optical axes as $\Pi_{p}$. As illustrated by Fig. 3, suppose also that the Ronchi grating is placed so that the

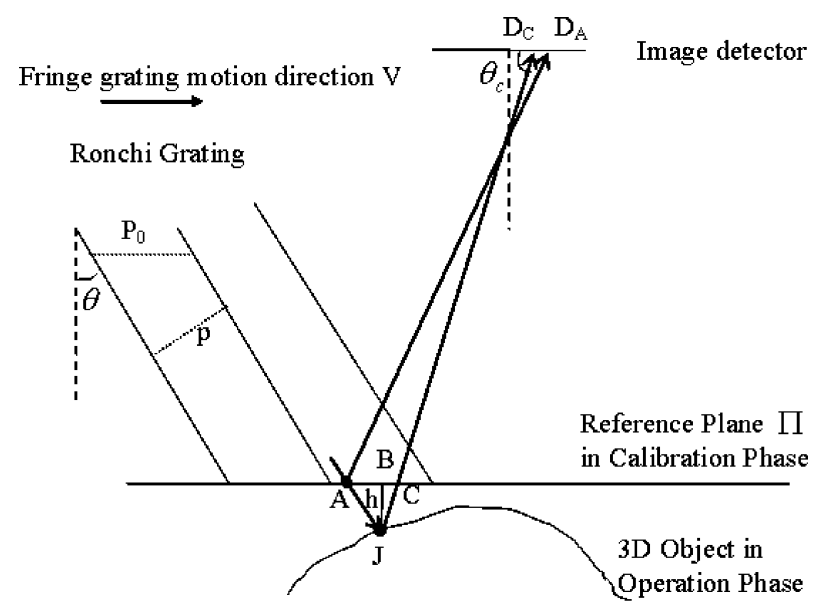

Fig. 3. Geometry setting of our binary projection system.

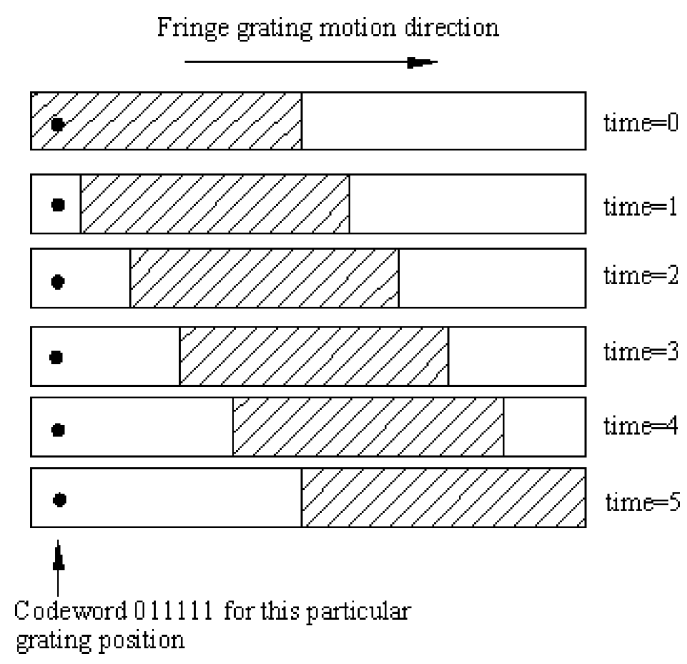

Fig. 4. Example of codeword production mechanism.

grating plane is parallel to the line $V$ that joins the camera's optical center and the light source (the line $v$ is analogous with the baseline in bicamera systems), that the fringes are in a direction that is perpendicular to plane $\Pi_{p}$, and that the physical motion of the fringe grating in space is in the direction of $V$. When pattern is projected onto the target surface, the surface will appear to have some bright or dark zones.

By shifting the grating a number of times, each time having a new image of the target surface captured using the camera, a binary codeword is developed for each image position over the sequence of captured images. The image position corresponds to a particular position on the target surface and in turn a particular position on the grating plane, and the associated codeword is readable from both the image data and the Ronchi grating. Fig. 4 illustrates the codeword of a particular position on the grating, where a dark fringe corresponds to a " 0 " and a bright fringe a "1."

Positions on the same fringe will have exactly the same codeword, so the fringe grating motion alone only induces line-to-contour correspondences between the grating plane and the image plane. However, correspondence ambiguity within each fringe could be resolved by the use of the epipolar 

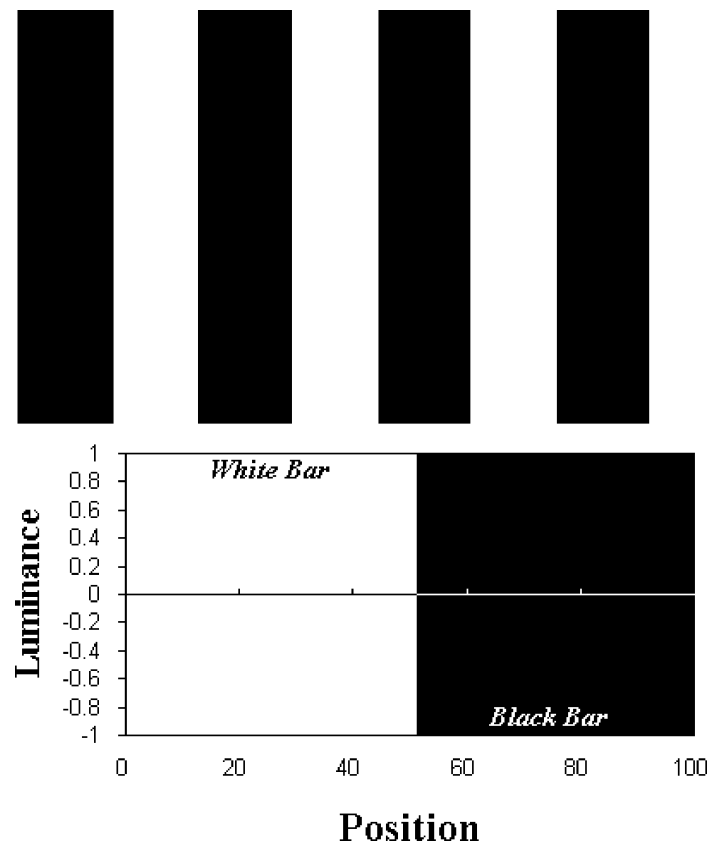

Fig. 5. Graph of the luminance of a binary grating.

geometry [26] that exists over the camera and the illumination source (on the epipolar geometry, the illumination source is to be regarded as the optical center of a second camera, and the fringe grating plane as the image plane of that camera). By the use of both the codeword and the epipolar geometry, point-to-point correspondences between the grating plane and image plane could readily be established.

\section{B. Image Data Binarization}

A binary pattern (stripe grating) is a repeating sequence of light and dark bars, with one pair of adjacent light and dark bars making up each cycle. These cycles repeat in a grating. One would expect that the step-like signals in the grating are reducible in the captured image data to a set of sharp edges like the bars shown in Fig. 5.

A binary pattern $f(x)$ of period $2 L$ can be expressed by a Fourier series:

$$
f(x)=\frac{4}{\pi} \sum_{n=1,3,5, \ldots}^{\infty} \frac{1}{n} \sin \left(\frac{n \pi x}{L}\right) .
$$

It consists of more high-frequency components than the sinusoidal grating, and the amplitudes of the components get smaller as the frequencies go up. In reality, what contrasts with Fig. 5 is, when light passes through the binary grating and is projected to a target object through a series of projection lenses, the high-order harmonics of the pattern are often diminished because of the lens system's limited bandwidth. Therefore, fringes projected on the target surface generally do not remain exactly black or white, but are changed to gray-level ones. In other words, the boundaries of the binary stripes will appear blurred in the captured image data, as illustrated by Fig. 6 .

However, the reconstruction mechanism is based upon discrete processing, and thus the detection of the fringes' boundaries and thereby the binarizaton of the image data is an impor-

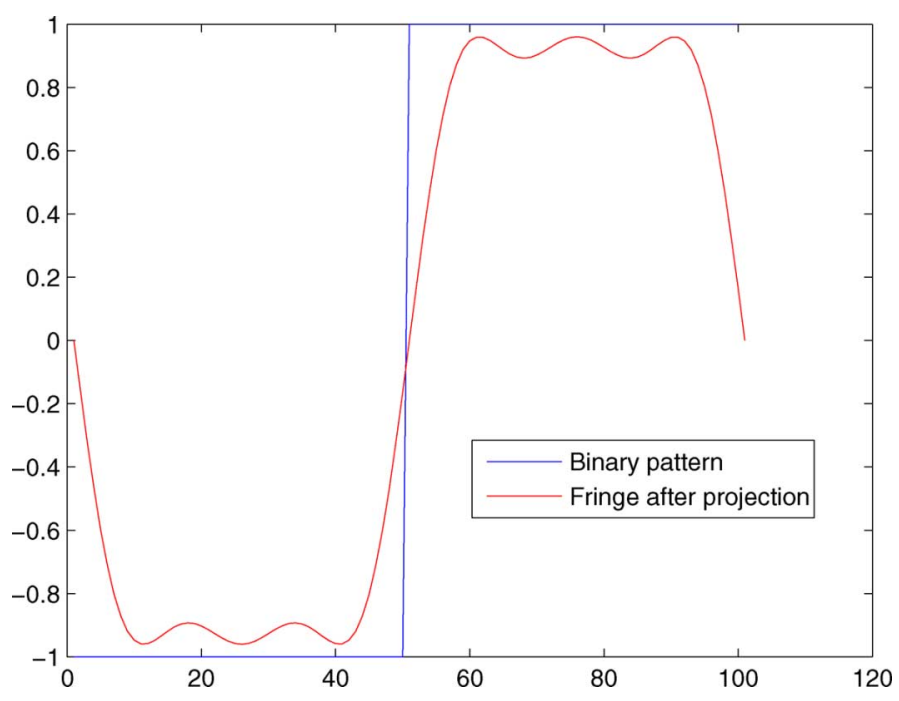

Fig. 6. Fringe pattern after the low-pass effect of lens projection. The fringe deforms because the high-order harmonics have their amplitudes diminished.

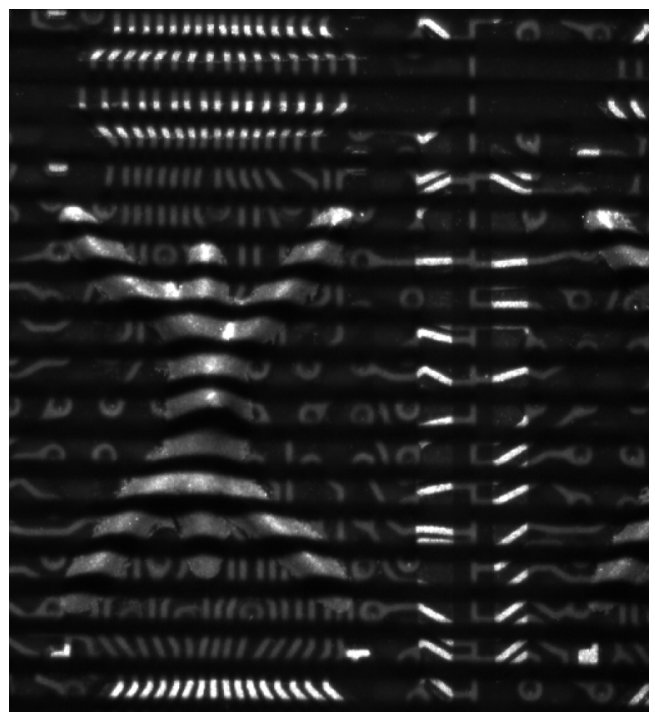

Fig. 7. Image captured by projecting a regular pattern onto a PCB surface.

tant step. Yet, different compositions of the target surface, some metallic (the solder surface) and some not (the substrate surface of the wafer), have different reflectance functions that contain both the specular and lambertian components. This makes fringe boundary detection in the image data a nontrivial task.

An often-used approach of converting a gray image into binary image is to adopt a global intensity threshold that is extracted based upon the intensity histogram property of the image: the binary image $B[i, j]$ is obtained from the gray-level image $I[i, j]$ using a cutoff intensity threshold $T$ in the following way:

$$
B[i, j]= \begin{cases}1, & \text { if } \mathrm{I}[\mathrm{i}, \mathrm{j}] \leq \mathrm{T} \\ 0, & \text { otherwise }\end{cases}
$$

We have tested this familiar approach in converting a graylevel image, captured by projecting a regular pattern onto a printed circuit board (PCB) surface (as shown in Fig. 7) into a binary image. The intensity histogram of the image is as shown 


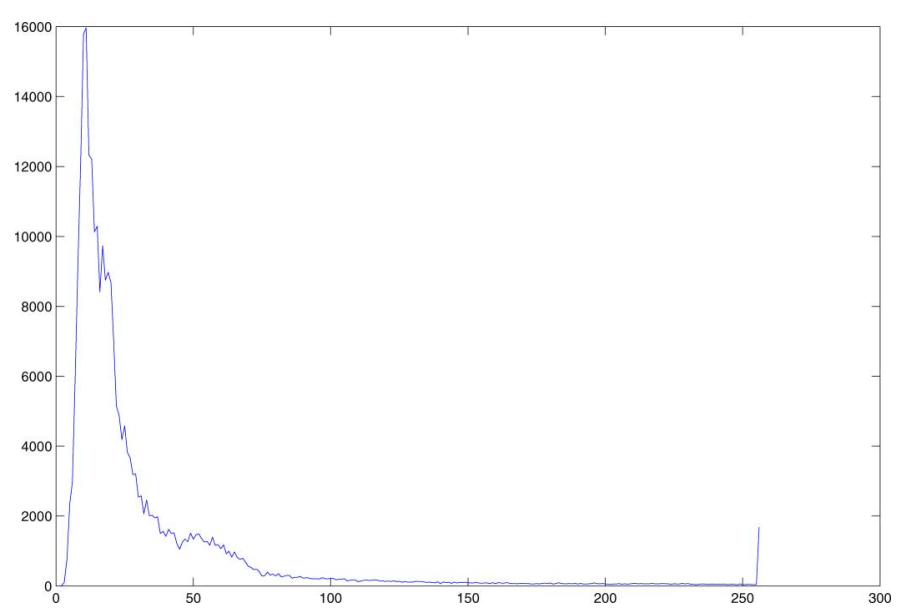

Fig. 8. Intensity histogram of the PCB image.

in Fig. 8. From the histogram, it can be observed that most intensities are less than 50 . Two peaks can be obtained from the histogram. The two peaks' positions are $I=11$ and $I=255$, respectively. Therefore, based on these two peaks, the threshold can be set to be between these two peaks. If the global threshold is set to be $T=133$, the binary image obtained will be as shown in Fig. 9(a). It is apparent in this image that most regions are unfortunately set to be "dark"; only a few regions with strong reflectance such as those from the metallic parts can appear "bright." If we set the global threshold as $T=45$, the binary image will be as shown in Fig. 9(b). Even though in this case the threshold is set to be rather low, some regions that should be regarded as "bright" are still classified as "dark," making the projected fringes on the inspected surface appear discontinuous. The example shows that the use of a global intensity threshold in binarizing the image data is not suitable in our case. This matters as well upon how accurately the edges can be located in the image, as upon how they can be located without their positions shifted due to variation in the reflectance function of the inspected surface. The above example shows that for images of our task, the edge locations can be dramatically shifted from the true ones by the reflectance variation of the illuminated surface.

Herein we describe our solution to the problem. It makes use of the spatial-temporal image volume for the target surface, as opposed to merely spatial derivatives of the image data as practiced traditionally, to tackle the issue of inhomogeneity in the reflectance function.

Consider any image position $(x, y)$ (say, position $D_{C}$ in Fig. 3) that perceives signal from a particular surface point $J$ of the target object; the surface point $J$ reflects light that goes through a particular position of the binary fringe grating plane. In the pattern projection process, the surface property (shape, reflectance, etc.) of the target object at surface point $J$ is invariant to the change of pattern. The invariance can be modeled as a transfer function $F_{S}(x, y)$. Similarly, the effect of the optical projection lens can be modeled as $F_{L}(x, y)$, and the effect of the image capture at the camera as $F_{C}$ (which is largely independent of the image position $(x, y))$. When a pattern $P$ is

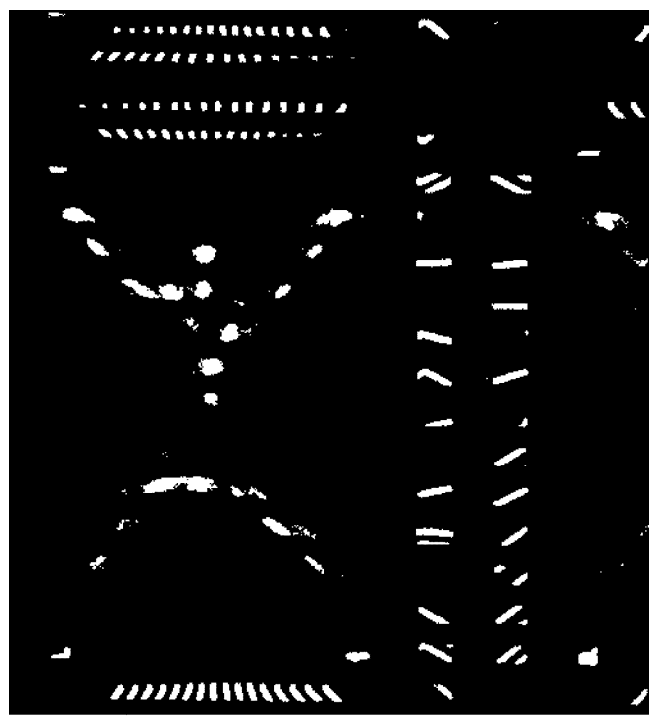

(a)

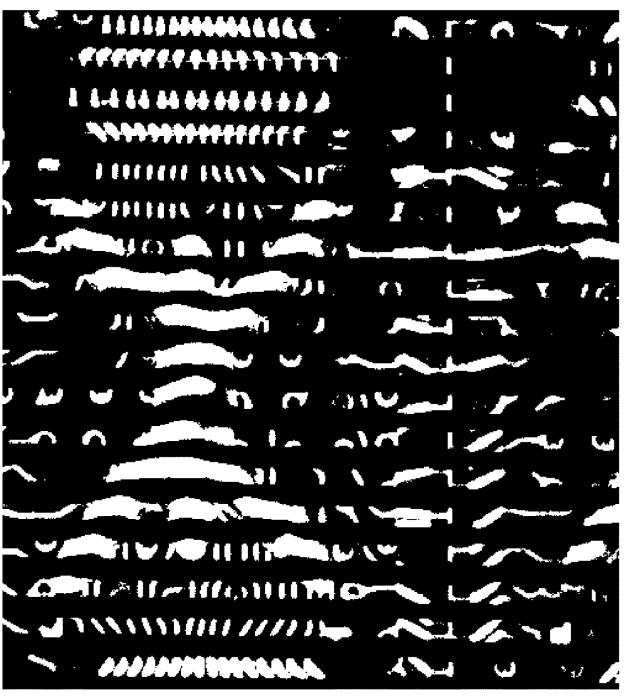

(b)

Fig. 9. Image binarized by the use of a global intensity threshold $T$. (a) $T=$ 133. (b) $T=45$.

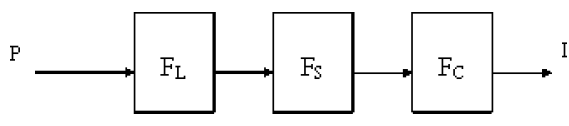

Fig. 10. Imaging model.

projected onto the surface, the image intensity $I(x, y)$ captured by the camera at position $(x, y)$ could be expressed as

$$
I(x, y)=F_{C} F_{S}(x, y) F_{L}(x, y) P(x, y)
$$

where $F_{L}(x, y), F_{S}(x, y)$, and $F_{C}$ are unknown but invariant (Fig. 10) to pattern $P$.

For a fixed binary grating, shifting it in space in small displacements will produce a series of patterns $P_{i}(x, y)$ 's for every 
image position $(x, y)$ on the image data. With $P_{i}(x, y)$ 's, different image intensities $I_{i}(x, y)$ 's can be obtained respectively as

$$
\left\{\begin{array}{l}
I_{1}(x, y)=F_{C} F_{S}(x, y) F_{L}(x, y) P_{1}(x, y) \\
I_{2}(x, y)=F_{C} F_{S}(x, y) F_{L}(x, y) P_{2}(x, y) \\
\vdots \\
I_{n}(x, y)=F_{C} F_{S}(x, y) F_{L}(x, y) P_{n}(x, y)
\end{array}\right.
$$

where $F_{S}(x, y)$ for image position $(x, y)$ represents the transfer function there on image intensity due to the illuminated surface.

Suppose that before we take data for the target object, we place a calibration plane $\Pi$ under exactly the same system to capture similar image data. The calibration plane $\Pi$ is a planar object. For each position $(x, y)$ in the image of the target object, there is a corresponding position $\left(x_{\Pi}, y_{\Pi}\right)$ in the reference image of the calibration plane $\Pi$, which receives signal through the same position of the binary fringe pattern. Image position $(x, y)$ to image position $\left(x_{\Pi}, y_{\Pi}\right)$ is as point $D_{C}$ to point $D_{A}$ in Fig. 3 .

As the binary grating shifts to create a series of patterns $P_{i}(x, y)$ 's at position $(x, y)$, like the intensity values $I_{i}(x, y)$ 's captured for the target object, image intensity values $J_{i}\left(x_{\Pi}, y_{\Pi}\right)$ 's are collected for the calibration plane $\Pi$

$$
\left\{\begin{array}{l}
J_{1}\left(x_{\Pi}, y_{\Pi}\right)=F_{C} F_{\Pi}\left(x_{\Pi}, y_{\Pi}\right) F_{L}(x, y) P_{1}(x, y) \\
J_{2}\left(x_{\Pi}, y_{\Pi}\right)=F_{C} F_{\Pi}\left(x_{\Pi}, y_{\Pi}\right) F_{L}(x, y) P_{2}(x, y) \\
\vdots \\
J_{n}\left(x_{\Pi}, y_{\Pi}\right)=F_{C} F_{\Pi}\left(x_{\Pi}, y_{\Pi}\right) F_{L}(x, y) P_{n}(x, y)
\end{array}\right.
$$

where $F_{\Pi}\left(x_{\Pi}, y_{\Pi}\right)$ represents the transfer function due to the calibration plane $\Pi$ for image position $\left(x_{P i}, y_{\Pi}\right)$.

Combining (3) and (4), we have $I_{i}(x, y) / J_{i}\left(x_{\Pi}, y_{\Pi}\right)=$ $F_{S}(x, y) / F_{\Pi}\left(x_{\Pi}, y_{\Pi}\right)$ which is a constant for all $i$, and therefore allows $I_{i}(x, y)$ 's be related to $J_{i}\left(x_{\Pi}, y_{\Pi}\right)$ 's without involving the unknowns $F_{L}(x, y), F_{S}(x, y), F_{\Pi}\left(x_{\Pi}, y_{\Pi}\right)$, and $F_{C}$ by

$$
\begin{aligned}
I_{1}(x, y): & I_{2}(x, y): \cdots: I_{n}(x, y) \\
& =J_{1}\left(x_{\Pi}, y_{\Pi}\right): J_{2}\left(x_{\Pi}, y_{\Pi}\right): \cdots: J_{n}\left(x_{\Pi}, y_{\Pi}\right) .
\end{aligned}
$$

Equation (5) shows that, in terms of the intensity's scale ratio, $I_{i}(x, y)$ 's and $J_{i}\left(x_{\Pi}, y_{\Pi}\right)$ 's display exactly the same profile (including 0 -to- 1 and 1 -to-0 transitions) along the time-axis. We shall term this the intensity scale-ratio resemblance property. With this property, the image frames at which position $(x, y)$ is a 0 -to-1 or 1-to-0 boundary point in $I$ (due to shifting of the binary grating in space) could be read from $J_{i}\left(x_{\Pi}, y_{\Pi}\right)$ 's. The only problem is, it seems correspondence between $(x, y)$ of $I$ and $\left(x_{\Pi}, y_{\Pi}\right)$ of $J$ needs be first established.

A parallel note regarding the image data of the calibration plane $\Pi$ is the following. Because of the plane's homogeneity in surface reflectance and planarity in shape, and of our adoption of the telecentric camera lens and projection lens in the system (so that both the pattern projection and the image capture are almost parallel), the time profile $J_{i}\left(x_{\Pi}, y_{\Pi}\right)$ 's of image intensities for the calibration plane has the same dynamics (though with generally different phases) irrespective of the image position $\left(x_{\Pi}, y_{\Pi}\right)$. In fact, due to the optical setup and the planar nature of the calibration plane, the dynamics at all image positions consist of only simple periodic cycles of 0-to-1 and 1-to-0 transitions in the same scale-range. We shall term this the homogeneous intensity scale-range property of the calibration data. With this property, one could easily determine from the data the intensity threshold (relative to the intensity fluctuation amplitude which is the same across all image positions) for pinpointing 0 -to- 1 and 1-to-0 transitions in the intensity profile of any image position $\left(x_{\Pi}, y_{\Pi}\right)$.

It follows that the correspondence between $(x, y)$ on $I$ and $\left(x_{\Pi}, y_{\Pi}\right)$ on $J$ is not necessary after all. By the use of the intensity homogeneous scale-range property of the calibration data, we can determine what the intensity threshold is for extracting the boundary points in $J$, which is the same across all image positions $\left(x_{\Pi}, y_{\Pi}\right)$ in the calibration data $J$. Since the scaleranges of all image positions in $J$ are the same, we could translate this absolute threshold to a global threshold on scale-ratio. By the use of the other property-the intensity scale-ratio resemblance between the target object data and the calibration data - we could borrow the above intensity scale-ratio threshold to detect boundary points in $I$, irrespective of the image position $(x, y)$.

In more detail, we first locate the edge positions (0-to- 1 and 1-to-0 jumps in the image data) in $J$. On this we could simply use traditional gradient-based edge detector, for the calibration plane is of homogeneous reflectance. Knowing the intensity value $J_{\text {edge }}$ of such edge point, we could determine the relative intensity threshold $T_{\text {edge }}$ (relative to the intensity fluctuation amplitude) as

$$
T_{\text {edge }}=\frac{J_{\text {edge }}-\min \left(J_{i}\right)}{\max \left(J_{i}\right)-\min \left(J_{i}\right)} .
$$

The relative threshold $T_{\text {edge }}$ can be applied to detect edge points in the image data of the target object. For any arbitrary position $(x, y)$ in image data $I$ of the target object, a number of intensities $I_{1}(x, y), I_{2}(x, y), \ldots, I_{n}(x, y)$ are available. $(x, y)$ is regarded as an edge point in any $k$ th image frame $I_{k}$ if the parameter $T_{k}$ computed as

$$
T_{k}=\frac{I_{k}-\min \left(I_{i}\right)}{\max \left(I_{i}\right)-\min \left(I_{i}\right)}
$$

is such that the quantity $\left|T_{k}-T_{\text {edge }}\right|$ is close enough to 0 .

Our fringe boundary detection mechanism puts together the whole sequence of image data to eliminate the influence of the projection lens, the camera, and the inhomogeneity of surface reflectance of the target object. Once the boundary information is acquired, the gray-level image data can be readily converted to binary data.

\section{3-D Measurement Without Explicit Triangulation}

Consider the system geometry shown in Fig. 3, where a collimated Ronchi pattern of period $p$ is projected, at an offset angle $\theta$ from the camera's optical axis, onto first a reference plane $\Pi$ and then later a 3-D object, where, for every image position $(x, y)$, the object shape $h(x, y)$ measured from the reference plane is desired. Here, the reference plane $\Pi$ is positioned 
approximately perpendicular to plane $\Pi_{P}$-the plane that contains the optical axes of both the camera and the illumination. All 3-D measurements at the end will be made against this reference plane.

As illustrated in Fig. 3, suppose $D_{A}$ is the pixel that measures the image information associated with point $A$ on the reference plane $\Pi$. If it is not the reference plane but the inspected surface that is illuminated, the same projection ray will hit upon a different surface point in 3-D, say point $J$ on the inspected surface, and be reflected to a different pixel on the image plane. Suppose we refer to that image pixel as $D_{C}$, and we refer to it that way because should it be the reference plane that is under illumination that particular image pixel will come from point $C$ of the reference plane. Notice that the binary information sensed at $D_{C}$ in the image data of the inspected surface is the same as that sensed at $D_{A}$ in the image data of the reference plane, owing to the same projection ray with which the two are associated. It then follows that the correspondence between $D_{C}$ and $D_{A}$ could be established by simply observing the Ronchi patterns at the two image positions in the reference plane image data and the object image data, respectively.

Suppose $A$ and $J$ are projections of the same Ronchi pattern on the reference plane and 3-D object, respectively. Suppose that $A$ is in the $i$ th column of the reference image (i.e., detector $D_{A}$ is in the $i$ th column of the camera's CCD array), and $J$ is in the $j$ th column of the object image (i.e., detector $D_{C}$ is in the $j$ th column of the CCD array). Suppose also that the CCD resolution is $R_{\mathrm{CCD}}$ (in terms of $\mu \mathrm{m} / \mathrm{pixel}$ ) and the magnification of the imaging system is $M$ (in terms of magnified times). The distance $A C$ can be determined from the separation of $i$ and $j$ in the image plane as

$$
A C=|i-j| \frac{R_{\mathrm{CCD}}}{M} .
$$

In turn, $A C$ is related to the surface height $h=B J$ by

$$
h=B J=\frac{A C}{\left(\tan \theta+\cot \theta_{c}\right)}=\frac{|i-j| \frac{R_{\mathrm{CCD}}}{M}}{\left(\tan \theta+\cot \theta_{c}\right)}
$$

where the angles $\theta$ and $\theta_{c}$ are as shown in Fig. 3. In practice, since the image sensing array is very small compared with the imaging distance and could be adjusted to have an orientation almost parallel to the reference plane, $\theta_{c}$ is nearly $90^{\circ}$. The above relation could thus be simplified to

$$
h=B J=\frac{A C}{\cot \theta}=|i-j| \frac{R_{\mathrm{CCD}}}{M} \cot \theta .
$$

We use the above equation to obtain depth, bypassing explicit triangulation by the use of a reference plane.

\section{EXPERIMENTS}

To illustrate the performance of the proposed approach on reflectance-inhomogeneous microsurfaces of hemispheric shape (the wafer bump array) and otherwise (the free-form shapes), we have performed experiments on a variety of objects. Here, we show the result on a micro PCB which contains tiny solder surfaces that are only $100 \sim 400 \mu \mathrm{m}$ tall and of all kinds of shapes. We also show the result on a typical wafer which has the

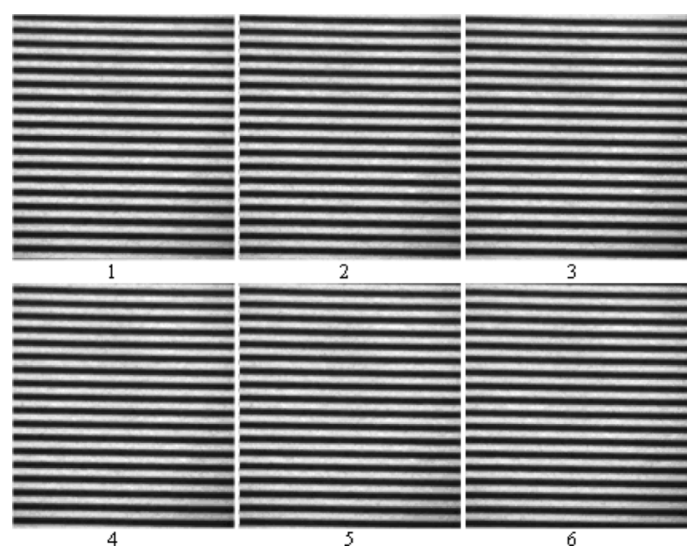

Fig. 11. Image sequence of the reference plane in the calibration phase under Ronchi pattern projection. These images were captured by shifting the pattern in steps of one-tenth of the pattern period.

traditional hemispheric solder bumps, with comparison with the result of the sinusoidal pattern projection approach.

\section{A. System Setup}

Calibration needs be done first to ensure that the illumination, the imaging, the grating plane, and the grating motion are aligned properly. The optical axes of the camera and illumination should be on the same plane $\Pi_{P}$. The Ronchi grating should have its plane parallel to the line $L$ that joins the camera's optical center and the light source, its fringes' direction perpendicular to plane $\Pi_{P}$, and its motion in the direction of $L$. It is also required that the reference plane be positioned so that it is perpendicular to plane $\Pi_{P}$.

A Ronchi grating with period $200 \mu \mathrm{m}$ and an incident angle $\theta=30^{\circ}$ of projection was adopted. The projection system consisted of an illuminator and several sets of lenses. The grating was shifted in space five times, each time by $20 \mu \mathrm{m}$. The magnification of the projection system was three times, which means the projected pattern would be enlarged three times to have a period of $600 \mu \mathrm{m}$ on the inspected surface. The imaging system had a magnification of 0.75 times, a resolution of $1000 \times 1000$, and a pixel size of $7.4 \mu \mathrm{m} \times 7.4 \mu \mathrm{m}$ in the CCD sensing array.

\section{B. Experiment on $P C B$}

In the calibration phase, we projected the fringe pattern onto a sheet of white paper, which served as the reference plane $\Pi$. By this step, codewords for various positions on the grating plane were transferred to the reference plane $\Pi$. Depth determination processing could thus be transferred from over the grating-and-image-plane-pair to over the reference-and-imageplane-pair through the use of (10) above. The image sequence for the reference plane is shown in Fig. 11.

In the operation phase, we projected the same fringe pattern onto a PCB, whose size was no bigger than $12 \mathrm{~mm} \times 12$ $\mathrm{mm} \times 600 \mu \mathrm{m}$. With five physical shifts of the pattern and image captures of the illuminated object, we established also a codeword for each point on the inspected surface. To put it simply, the codeword was the sequence of " 1 "s and " 0 "s in the image sequence (shown in Fig. 12) at every image position. 

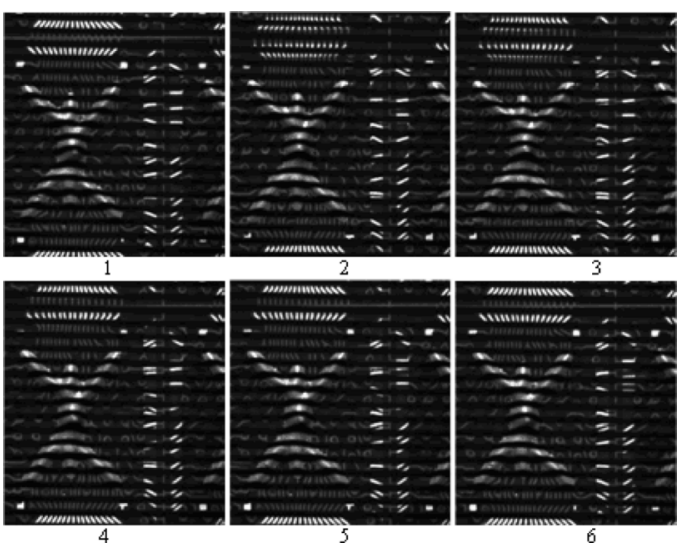

Fig. 12. Image sequence of the inspected surface (PCB) in the operation phase, under projection of the same pattern as in the previous figure. These images were captured by shifting the pattern in steps of one-tenth of the pattern period.
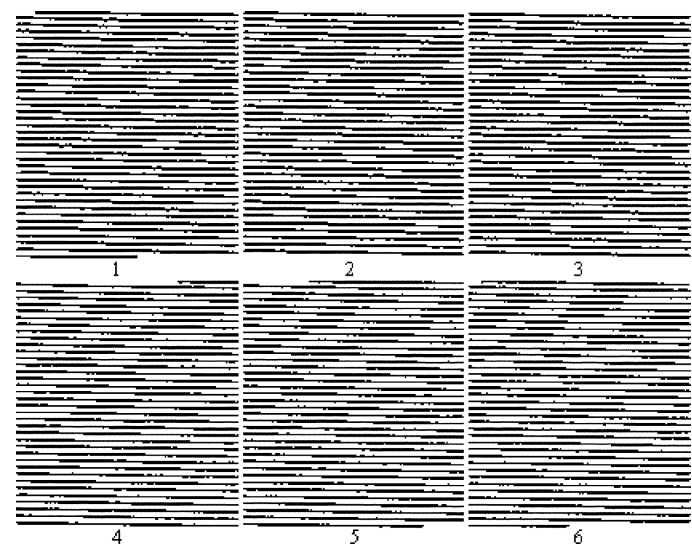

Fig. 13. Fringe boundary detection for the reference plane by a Canny edge detector.
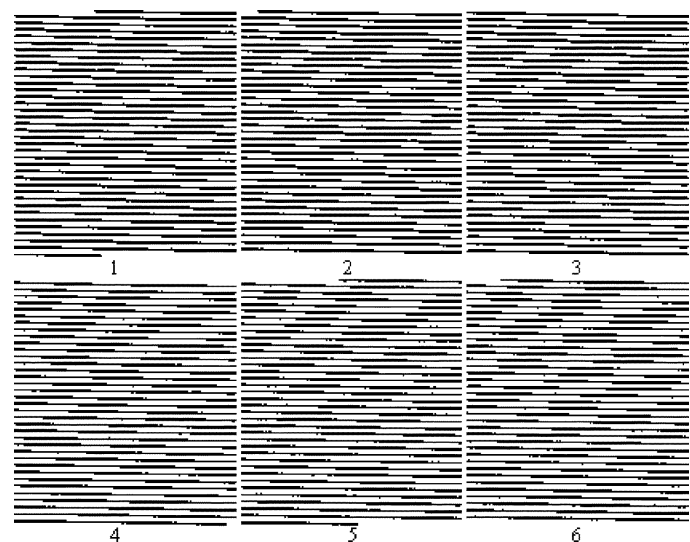

Fig. 14. Fringe boundary detection for the reference plane by our time profilebased edge detector.

1) Boundary Detection: In the calibration step, we projected the pattern onto a piece of white paper which possessed homogeneous reflectance function. For such reflectance-homogeneous surface, the fringe boundaries in the image data could be reliably located by traditional edge detection algorithms like the Canny edge detector (shown in Fig. 13). Based on the result on such a calibration surface, the threshold $T_{\text {edge }}$ as expressed by (6) was determined to be 0.497 , which would find its use in the

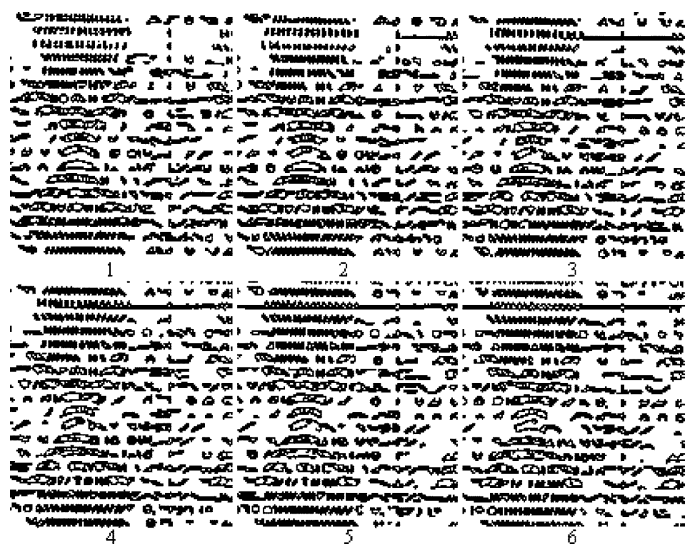

Fig. 15. Fringe boundary detection for the inspected surface by a Canny edge detector.

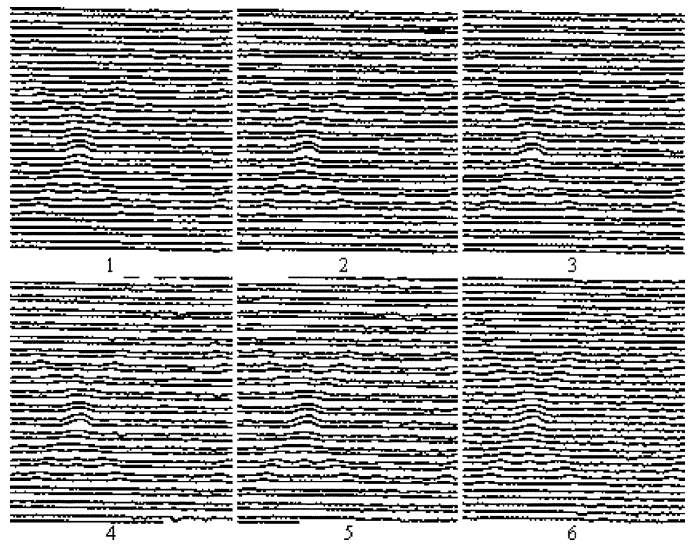

Fig. 16. Fringe boundary detection for the inspected surface by our time profile-based edge detector.

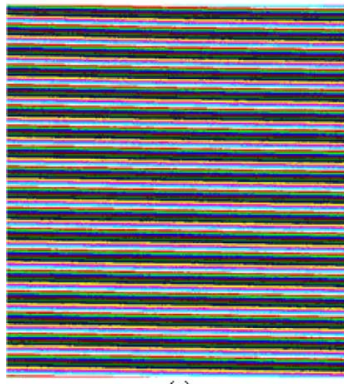

(a)

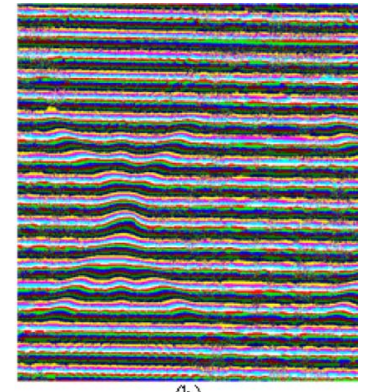

(b)
Fig. 17. Codeword maps for (a) the reference plane and (b) the inspected surface.

proposed time profile-based boundary detection mechanism. On edge detection for the calibration plane, the time profile-based boundary detection mechanism could also obtain good results (shown in Fig. 14). The two algorithms give similar results, demonstrating that the time profile-based approach could be as good as the image gradient-based approach on scenes with homogeneous reflectance.

We did the same comparison on the image data of the operation phase. Here, the inspected surface was made of different materials including metallic solder and nonmetallic substrate, which led to inhomogeneity of the surface reflectance function varying from the specular to the diffuse extremes. The image intensity distribution, so influenced by the surface reflectance, led to the deteriorated result of boundary detection under the 

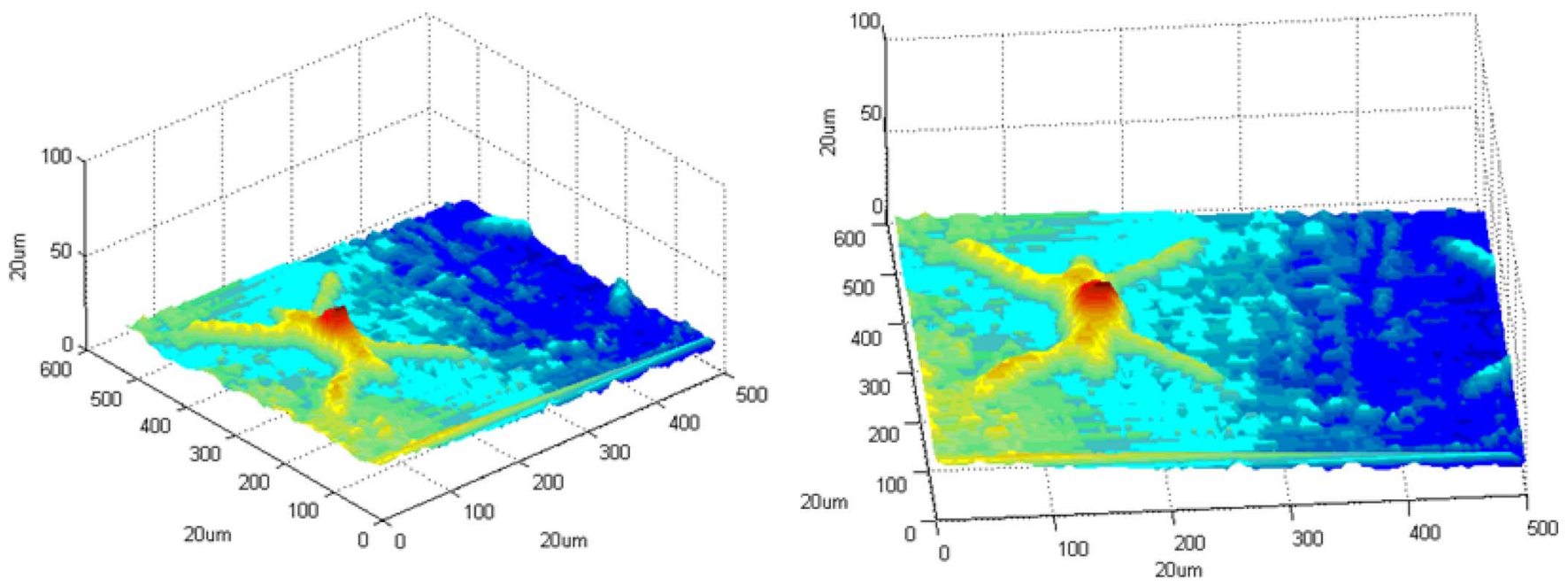

Fig. 18. 3-D reconstruction of the inspected surface by the proposed method, as observed from different angles.

Canny edge detector (as shown in Fig. 15). However, our time profile-based mechanism could reduce the influence of the reflectance function inhomogeneity and get a much better result (as shown in Fig. 16). The detected boundaries were found to be much closer to the expected ones on mapping them to the image sequence.

2) Result: By the use of the proposed boundary detection mechanism on the spatial-temporal volume of the associated image data, the gray-level images could be readily converted to a binary image. From the binarized image sequence, we obtained the codeword maps (shown in Fig. 17, where different colors represent different codewords) for the reference plane and the inspected surface, respectively. With the two codeword maps, corresponding point pairs in the two maps could be obtained by associating image points with the same sequence of "1"s and "0"s. With the correspondences, 3-D position or depth disparity could be estimated for each point of the inspected surface using the method outlined in Section II. Fig. 18 shows the 3-D reconstruction of the inspected surface.

We would like to point out that in our implementation we only consider the image points that are close to the edge of the Ronchi pattern in at least one of the captured images and attempt correspondence extraction and 3-D reconstruction at those points only. The reason is, for other (non-edge) places in the image, neighboring image positions might have similar or the same codeword due to the limited image resolution or the limited resolution of the fringe motion, and that would compromise the accuracy in their 3-D reconstruction. We found from our experiments that image positions that are edge points in either one of the captured images constitute about $50 \%$ of all the image positions, and at such positions, depth could be recovered directly. As for the 3-D information of the other 50\% of the image positions, we suggest that they could be interpolated from those of the edge points.

\section{Experiment on Wafer Bumps}

We have also performed experiments on wafer bumps which were of diameters about $300 \mu \mathrm{m}$. The size of the wafer was no
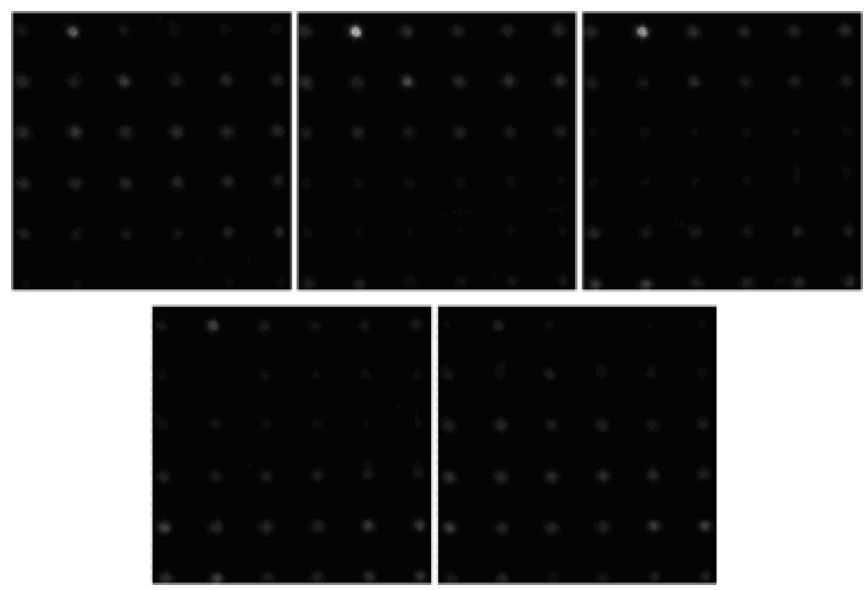

Fig. 19. Operation phase image sequence in projecting sinusoidal pattern onto the wafer. These images were captured by shifting the pattern in $2 \pi / 5$ increments.

bigger than $8 \mathrm{~mm} \times 8 \mathrm{~mm} \times 500 \mu \mathrm{m}$. First, we reconstructed the surface using sinusoidal pattern projection with phase shifting. A sinusoidal pattern with period $3 \mathrm{lp} / \mathrm{mm}$ (line pairs $/ \mathrm{mm}$ ) was projected onto the surface. Five fringes were detected by shifting the phase in $2 \pi / 5$ increments (shown in Fig. 19). The phase was then computed as [15]

$$
\phi=\arctan \frac{\sum_{n=1}^{5}\left(I_{n} \sin \frac{2 n \pi}{5}\right)}{\sum_{n=1}^{5}\left(I_{n} \cos \frac{2 n \pi}{5}\right)}
$$

With the phases for the reference plane and the inspected surface in hand, the 3-D structure of the inspected surface could be obtained. As the bump was mirror-like and the reflectance of the substrate was rather low, it was difficult to get the 3-D information of the bumps and substrate at the same time. Increasing the illumination would lead to image saturation on the bumps, yet the signal-to-noise ratio on the substrate surface was very low if illumination was not raised. Thus, in general, the substrate 


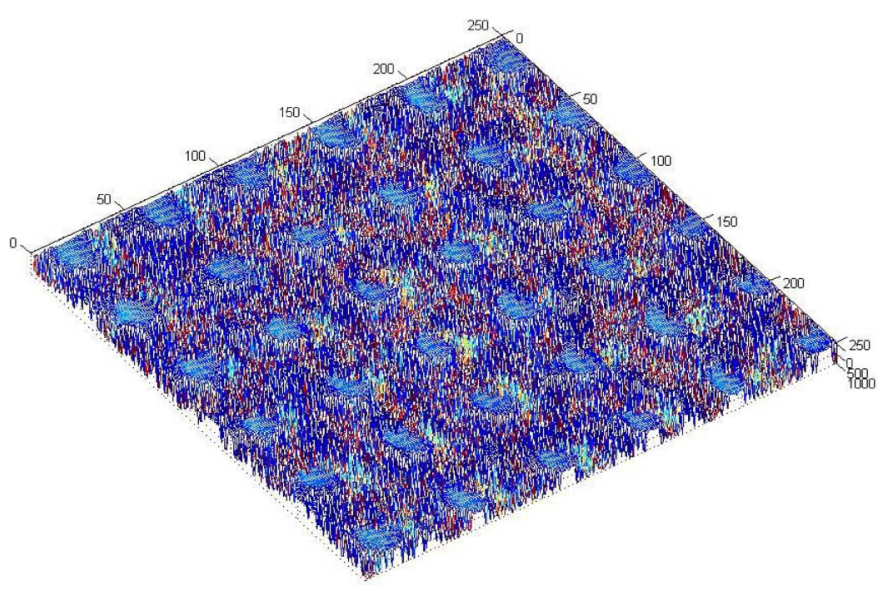

Fig. 20. 3-D reconstruction of the wafer (including the substrate surface) using sinusoidal pattern projection. The result appears noisy on the substrate, as the sinusoidal pattern projection is sensitive to noise especially in low-intensity regions.

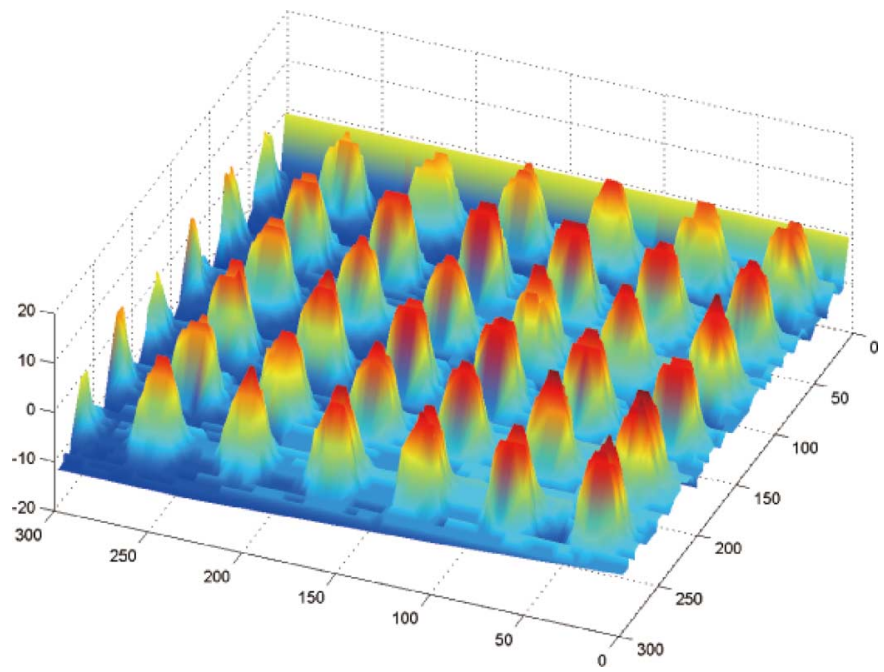

Fig. 21. 3-D reconstruction of wafer (the bumps and the substrate surface together) using binary pattern projection.

reconstruction was very sensitive to noise, as indicated by the result shown in Fig. 20.

We tested our approach on the same wafer for 3-D structure reconstruction (including the bumps and the substrate surface). We shifted the binary grating four times and took five images. It produced eight codewords, and the $Z$ resolution (i.e., the resolution in the direction perpendicular to the reference plane) could reach $20 \mu \mathrm{m}$. The result is shown in Fig. 21. The 3-D surface including the bumps and substrate was reconstructed to one that was observably close to the expected one. Putting Figs. 20 and 21 together, it could be observed that, especially on the substrate parts of the wafer, the reconstruction result from sinusoidal fringe projection was rather noisy and not as good as that from the proposed approach. We ascribe the better reconstruction quality of the proposed approach to the property that the approach is less affected by variation in the reflectance function of the target object. As the inspection task in our case involves wafer that consists of both metallic components (solder bumps)

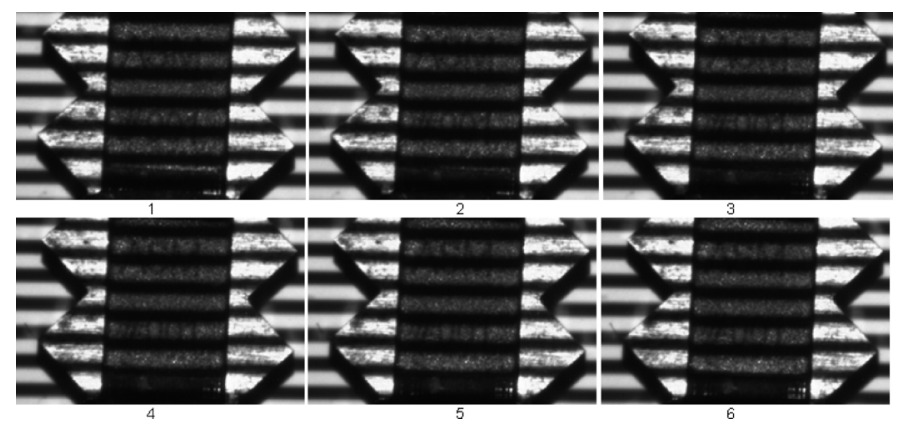

Fig. 22. Image sequence of a micromanufactured object with known surface profile.

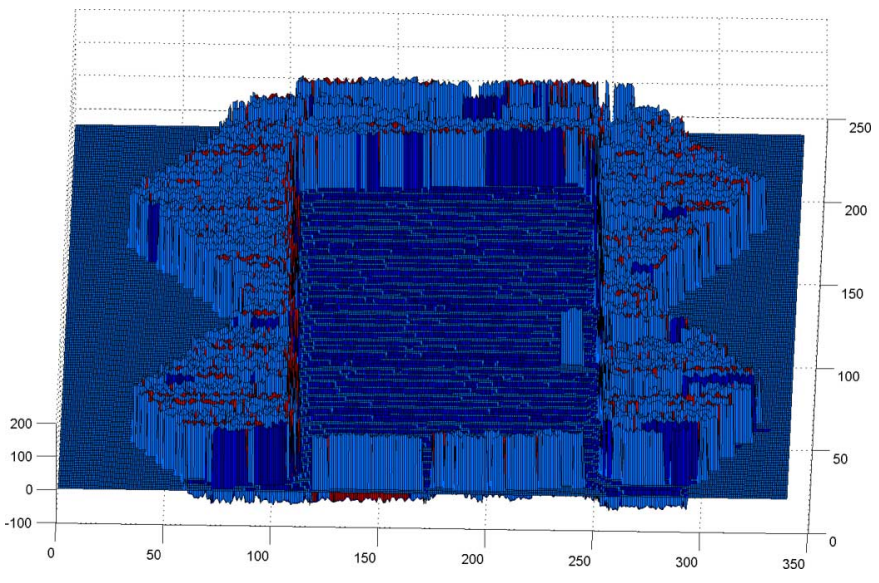

Fig. 23. Reconstruction result of the micromanufactured object.

as well as nonmetallic components (the substrate surface), we believe our approach has an edge on the task.

\section{Performance Evaluation}

What is inconvenient about real image experiments is that the ground truth of the associated object for evaluating solution quality is often difficult to obtain. It is especially so when the shapes of the target are of microscopic scale. To evaluate the 3-D reconstruction quality of the proposed mechanism, we made use of a micro-machined object whose shape was carefully crafted, and we regarded the intended shape as the ground truth. The micro-object was a rather simple object made of metal and composed of two planes. As shown in Fig. 22, the height of the central portion of the object was $200 \mu \mathrm{m}$ lower than that of the peripheral portion. The central portion had low reflectance, while the peripheral portion had high reflectance. Therefore, the reflectance of the micro-object was a variant like that of the PCB or wafer.

With six images of the object and six images of a reference plane, the 3-D shape of the object was reconstructed as shown in Fig. 23. Upon comparison with the ground truth, the reconstruction was found to have a mean square error of $9.43 \mu \mathrm{m}$. The experiment shows that the reconstruction quality of the proposed system is within reasonable bounds. We believe, with the use of higher resolution camera and higher frequency fringe pattern, the reconstruction accuracy and resolution could be further improved. 


\section{CONCLUSION AND FutURE Work}

A new design of the structured light sensing system has been presented. It projects binary pattern to the inspected surface by the use of a single light source and a fixed binary fringe grating, as opposed to the traditional use of an array of light sources. To equip each 3-D position with a distinct binary code, the pattern projection is varied by having the fringe grating shifted in space, and in each shifting step having the illuminated surface imaged. The system setup has a divergent configuration. It makes the size of the system much smaller than that of the traditional setup, and it better suited for the inspection of microscopic electronic components. The discrete nature of the system allows complicating issues like the specular nature of some metallic components in the target surface, image saturation, and imaging noise to be much reduced. In particular, two issues in the proposed design are addressed in detail in this paper, namely how the fringe boundary in the image data can be localized and how 3-D reconstruction can be achieved without explicit triangulation. Experiments showed that the design is effective in reconstructing microscopic objects like wafer bumps, and the reconstruction quality could be better than that of the existing approaches like the sinusoidal fringe projection approach, especially when dealing with objects of large variation in the reflectance function.

Future work includes optimal design of the fringe pattern and the shifting steps. In general, the fringe pattern needs not be periodic, and the shifting steps need not be uniform. Future work also includes the use of redundant binary coding to tackle the problem of multiple reflections.

\section{ACKNOWLEDGMENT}

The authors would like to thank Associate Editor I. Fidan for his prompt reviews and the reviewers for their invaluable comments.

\section{REFERENCES}

[1] H. Stern, "Laser Based 3-D Surface Mapping for Manufacturing Diagnostics and Reserve Engineering," in Proc. IEEE Conf. Aerospace Electron., 1992, pp. 1205-1212.

[2] S. Wang, B. Zhuang, and W. Zhang, "New principle of optical displacement measurement based on light scattering from rough surface," in Three-Dimensional and Laser-Based Systems for Metrology and Inspection II, K. Harding and D. Svetkoff, Eds. Bellingham, WA: SPIE, 1997, vol. 2909, pp. 37-42.

[3] M. Ishihara and H. Sasaki, "High-speed 3D shape measurement using a nonscanning multiple-beam confocal imaging system," in Laser Interferometry IX: Techniques and Analysis, M. Kujawinska, G. M. Brown, and M. Takeda, Eds. Bellingham, WA: SPIE, 1998, vol. 3478, , pp. $68-75$.

[4] A. Rao, N. Ramesh, F. Wu, J. Mandeville, and P. Kerstens, "Algorithms for a fast confocal optical inspection system," in Proc. IEEE Workshop Applicat. Comput. Vision, 1992, pp. 298-305.

[5] A. Schick and M. Kedziora, "Inspection and process evaluation for flip chip bumping and csp by scanning 3D confocal microscopy," in Proc. Adv. Packag. Mater., 2002, pp. 116-119.

[6] S. Bucourt, E. L. Falher, Y. Malet, and G. Sirat, "Conoscopic optical probes: A new generation of metrological instruments," in 1994 Eur. Conf. Lasers Electro-Optics, 1994, pp. 330-331.

[7] G. Y. Sirat and D. Psaltis, "Conoscodic holography," Opt. Lett., vol. 10, no. 1, pp. 4-6, 1985.
[8] H. Takasaki, "Moire topography," Appl. Opt., vol. 9, pp. 1467-1472, 1970.

[9] S. Kim, Y. Choi, and J. Oh, "Three-dimensional profile measurement of fine object by phase-shifting Moiré interferomety," in Three-Dimensional and Laser-Based Systems for Metrology and Inspection II, K. Harding and D. Svetkoff, Eds. Bellingham, WA: SPIE, 1997, vol. 2909, pp. 28-36.

[10] M. D. Altschuler, B. R. Altschuler, J. T. Dijak, L. A. Tamburino, and B. Woolford, "Robot vision by encoded light beams," in Three-Dimensional Machine Vision, T. Kanade, Ed. Dordrecht, The Netherlands: Kluwer, 1987, pp. 53-71.

[11] M. D. Altschuler, B. R. Altschuler, and J. Taboada, "Laser electro-optic system for rapid three-dimensional (3-D) topographic mapping of surfaces," Opt. Eng., vol. 20, no. 6, pp. 953-961, 1981.

[12] D. Bergmann, "New approach for automatic surface reconstruction with coded light," in Remote Sensing and Reconstruction for Three-Dimensional Objects and Scenes, T. Schenk, Ed. Bellingham, WA: SPIE, 1995, vol. 2572, pp. 2-9.

[13] D. Caspi, N. Kiryati, and J. Shamir, "Range imaging with adaptive color structured light," IEEE Trans. Pattern Anal. Mach. Intell., vol. 20, no. 5, pp. 470-480, May 1998.

[14] J. Gühring, "Dense 3-D surface acquisition by structured light using off-the-shelf components," in Videometrics and Optical Methods for 3D Shape Measurement, S. F. El-Hakim and A. Gruen, Eds. Bellingham, WA: SPIE, 2001, vol. 4309, pp. 220-231.

[15] M. Halioua and H. Liu, "Optical sensing techniques for 3-D machine vision," in Optical Techniques for Industrial Inspection, P. Cielo, Ed. Bellingham, WA: SPIE, 1986, vol. 665, pp. 150-161.

[16] O. Hall-Holt and S. Rusinkiewicz, "Stripe boundary codes for real-time structured-light range scanning of moving objects," in Proc. Int. Conf. Comput. Vision, 2001, pp. 359-366.

[17] P. S. Huang, C. P. Zhang, and F. P. Chiang, "High speed 3-D shape measurement based on digital fringe projection," Opt. Eng., vol. 42, no. 1, pp. 163-168, 2003.

[18] S. Inokuchi, K. Sato, and F. Matsuda, "Range-imaging for 3-D object recognition," in Proc. Int. Conf. Pattern Recognition, 1984, pp. 806-808.

[19] J. L. Posdamer and M. Altschuler, "Surface measurement by spaceencoded projected beam systems," Comput. Graphics Image Process., vol. 18 , no. 1, pp. 1-17, 1982.

[20] V. Srinivasan, H. Liu, and M. Halioua, "Automated phase-measuring profilometry of 3-D diffuse objects," Appl. Opt., vol. 23, pp. 3105-3108, 1984.

[21] C. Wust and D. W. Capson, "Surface profile measurement using color fringe projection," Mach. Vision Applicat., vol. 4, pp. 193-203, 1991.

[22] V. Srinivasan, H. Liu, and M. Halioua, "Automated phase-measuring profilometry: A phase mapping approach," Appl. Opt., vol. 24, pp. 185-188, 1985.

[23] J. Pan, P. S. Huang, and F.-P. Chiang, "Color-coded binary fringe projection technique for 3-D shape measurement," Opt. Eng., vol. 44, no. 2, p. 023606, 2005.

[24] J. Cheng, R. Chung, E. Lam, K. Fung, F. Wang, and W. Leung, "Threedimensional reconstruction of wafer solder bumps using binary pattern projection," in Machine Vision Applications in Industrial Inspection XIII, J. R. Price and F. Meriaudeau, Eds. Bellingham, WA: SPIE, 2005, vol. 5679, pp. 44-52.

[25] J. Cheng, R. Chung, and E. Lam, "Structured light-based 3D reconstruction for device of minute size," in Eur. Conf. Comput. Vision's 2nd Workshop Applicat. Comput. Vision, 2006, pp. 128-136.

[26] O. Faugeras, "Stratification of three-dimensional vision: Projective, affine, and metric representations," J. Opt. Soc. Amer.: A, vol. 12, pp. 465-484, 1995.

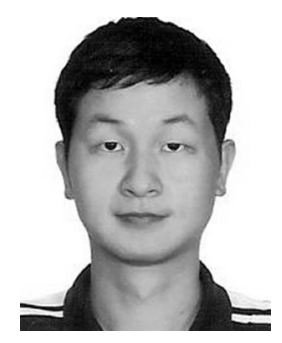

Jun Cheng received the B.Eng., B.Fin., and M.Eng. degrees from the University of Science and Technology of China, Beijing, in 1999 and 2002 respectively, and the $\mathrm{Ph} . \mathrm{D}$. degree from the Chinese University of Hong Kong in 2006.

Currently he is with the Shenzhen Institute of Advanced Integration Technology, Chinese Academy of Sciences/The Chinese University of Hong Kong, Shenzhen, as a Research Associate Fellow and Director of the Laboratory for Semiconductor Equipment. His research interests include computer vision, robotics, machine intelligence, and control. 


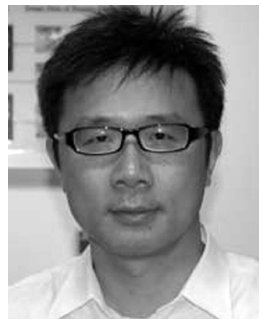

Chi-Kit Ronald Chung (SM'99) received the B.S.E.E. degree from the University of Hong Kong, Hong Kong, China, and the Ph.D. degree in computer engineering from University of Southern California, Los Angeles.

He was an Integrated Circuit Design Engineer and an Electronics Engineer in industry. He is currently with the Chinese University of Hong Kong as Director of the Computer Vision Laboratory and a Professor in the Department of Mechanical and Automation Engineering. His research interests include computer vision and robotics.

Prof. Chung is a Chartered Engineer of the Engineering Council of the U.K., and a member of MENSA. He was the Chairman of the IEEE Hong Kong Section Joint Chapter on Robotics and Automation Society and Control Systems Society in the years 2001-2003.

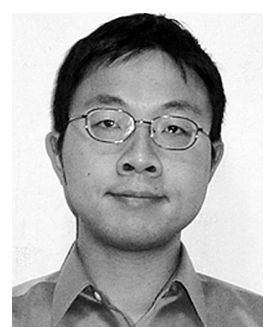

Edmund Y. Lam (SM'05) received the B.S., M.S., and $\mathrm{Ph} . \mathrm{D}$. degrees in electrical engineering from Stanford University, Stanford, CA, in 1995, 1996, and 2000, respectively.

At Stanford, he was a member of the Information Systems Laboratory, conducting research for the Stanford Programmable Digital Camera project. His focus was on developing image restoration algorithms for digital photography. Outside Stanford, he also consulted for industry in the areas of digital camera systems design and algorithms development. Before returning to academia, he worked in the Reticle and Photomask Inspection Division (RAPID), KLA-Tencor Corporation, San Jose, CA, as a Senior Engineer. His responsibility was to improve on the core die-to-die and die-to-database inspection algorithms, especially for phase shift masks. He is currently an Assistant Professor in the Department of Electrical and Electronic Engineering, University of Hong Kong. His research interests include image processing and computational imaging, particularly when applied to semiconductor manufacturing.

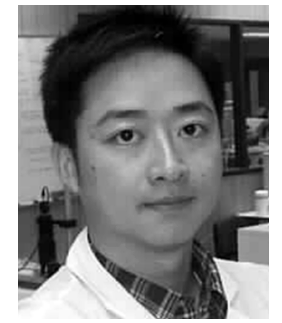

Kenneth S. M. Fung received the Ph.D. degree in electrical engineering from the University of Hong Kong, Hong Kong, China, in 1999.

$\mathrm{He}$ is the Technical Manager in the Research and Development Department, ASM Assembly Automation, Ltd., Hong Kong. After receiving the Ph.D. degree, he joined ASM Assembly Automation, Ltd., as a Senior Engineer. He developed a subpixel accuracy, high-speed and robust computer vision alignment algorithm that was applied in all ASM products and boosted the capability and vision technology level of the company's semiconductor packaging machines. Currently, he leads a team of research engineers responsible for the projects in machine vision inspection and algorithm development. He also provides technical supervision for a team of vision application engineers who are responsible for the projects in developing machine vision applications on ASM products. Kenneth's research interests are computer vision, pattern recognition, digital image processing, and artificial neural networks.

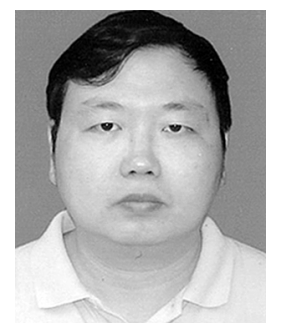

Fan Wang received the B.S. degree from Tianjin University, Tianjin, China, in 1985, the M.S. degree in physics from the South China University of Technology, Guangzhou, China, in 1988, and the $\mathrm{Ph} . \mathrm{D}$. degree from Tianjin University in 1991.

$\mathrm{He}$ is currently with the Research and Development Department, ASM Assembly Automation, Ltd., Hong Kong, China. He has published papers on optical association memory and optical information processing. His recent interests include optical projector and 3-D display technologies.

W. H. Leung is currently with the Research and Development Department, ASM Assembly Automation, Ltd., Hong Kong, China. 